\title{
Virulence Reversion in Staphylococcus aureus
}

\author{
Vishal Gor', Mitsuaki Hoshi'2, Aya J. Takemura ${ }^{3}$, Masato Higashide' ${ }^{2}$, Veronica Medrano \\ Romero $^{1}$, Ryosuke L. Ohniwa ${ }^{4,5}$ and Kazuya Morikawa ${ }^{4 *}$ \\ 1 Graduate School of Comprehensive Human Sciences, University of Tsukuba, Tsukuba, 305-8575, Japan \\ 2 Kotobiken Medical Laboratories, Inc., Kamiyokoba, Tsukuba 305-0584, Japan \\ 3 Human Biology Program, School of Integrative and Global Majors, University of Tsukuba, 305-8575, \\ Tsukuba, Japan \\ 4 Division of Biomedical Science, Faculty of Medicine, University of Tsukuba, Tsukuba 305-8575, Japan \\ 5 Center for Biotechnology, National Taiwan University, Taipei, Taiwan \\ *Corresponding author e-mail address: morikawa.kazuya.ga@u.tsukuba.ac.jp \\ + Presented at the 1st International Electronic Conference on Microbiology, 2-30 November 2020; Available \\ online: https://ecm2020.sciforum.net/
}

Published: 2 November 2020

\begin{abstract}
Staphylococcus aureus is a Gram-positive opportunistic pathogen that imposes a heavy burden on society. What sets this pathogen apart is the sheer spectrum of infections it can cause, which range from benign skin and soft tissue infections to lethal endocarditis and bacteraemia. The ability of S. aureus to cause this gamut of infections is conferred by its arsenal of virulence factors that are under the control of the Accessory Gene Regulator (Agr) system. However, a large proportion of clinical isolates have inactivating mutations in this important regulatory system. We previously showed that, contrary to the common dogma, not all these mutations are evolutionary 'dead-ends' and a fraction are phase variants which can revert to an Agr active state. Here we report that some Agr deficient isolates can revert a haemolytic phenotype without repairing their Agr system. We collected as series of $30 \mathrm{Agr}$ negative primary patient samples in order to assess the significance of our previous findings on the existence of Agr phase variants. We used primary samples to avoid strains that had undergone multiple clonal expansions before being tested for reversibility. We assessed Agr reversibility by serially passaging strains and screening for phenotypic reversion of haemolysis. We show that two strains reverted haemolysis and one reverted alpha haemolysin activity without any genetic changes in agr (and hla for the alpha revertant). These results add further complexity to the phenomenon of Agr shutdown observed in the clinical setting and corroborate recent findings of compensatory mutations arising in Agr deficient clinical strains.
\end{abstract}

Keywords: Staphylococcus aureus; virulence; Agr dysfunction

\section{Introduction}

Staphylococcus aureus is a clinically important opportunistic pathogen that merits attention due to the diversity and severity of infections that it causes [1]. Its success as a pathogen is facilitated by its gamut of virulence factors [2], whose expression must be finely tuned to control the transition of S. aureus' lifestyle from a commensal or chronic state to an aggressively invasive one. The responsibility for providing this balance falls to the global virulence regulatory system, the Accessory Gene Regulator (Agr) system. Briefly, the Agr system consists of two divergently transcribed loci that encode for a quorum sensing circuit and a regulatory RNA gene [3]. Cells produce a basal level of an autoinducing pheromone encoded in the quorum sensing circuit and when the extracellular concentration of this pheromone surpasses a threshold the cell detects it and transcribes the regulatory RNA, RNAIII, in response. RNAIII de-represses the translation of Staphylococcus aureus 
virulence factors associated with invasive and aggressive infections and represses translation of factors associated with a chronic or commensal lifestyle [4].

Interestingly, although the importance of the Agr system in establishing infection has been robustly demonstrated, there are numerous reports of Agr negative mutants being isolated from the clinical setting [5-7]. The previous dogma was that these strains have no evolutionary future and are fated to go to extinct, hence they have been referred to as 'dead-end' mutants.

In bacteria, there exists a phenomenon referred to as 'Phase Variation' that is an evolutionarily sustainable mechanisms of gene expression switching [8]. We recently demonstrated that some Agr negative strains are Phase Variants and not 'dead-end' mutants [9]. These strains could revert their Agr negative phenotype with underlying phase variation mechanisms. Importantly, we identified a single reversible Agr negative clinical isolate that was a Phase Variant. However, our collection of clinical strains had undergone several clonal expansions and so the true significance of our findings on the clinical scale could not be assessed.

In this study, we aimed to clarify the prevalence of Agr Phase Variants in the clinical setting. We collected a series of primary isolates that were directly form the patients and had not undergone clonal expansion and tested the reversibility of their Agr status. While we could not identify any Phase Variants, we did notice that two strains were able to revert their haemolytic phenotype on without any change in their Agr status. Interestingly, an additional strain showed clear reversion of alpha haemolysin, and important virulence factor for S. aureus, without any change in Agr, which is the direct controller for hla expression. These results add a further layer to the understanding of Agr deficiency of $S$. aureus in the clinical scene.

\section{Materials and Methods}

\subsection{Clinical strain collection}

A series of swabs from infection sites of patients confirmed to be infected with $S$. aureus were gathered from the Kanto region of Japan. Swabs were streaked on Sheep Blood Agar (SBA) and single non-haemolytic colonies were isolated to make glycerol stocks. Stocks were used to assess the Agr status of the strains using the method described below.

\subsection{Assessment of Agr activity on Sheep Blood Agar Plates}

A CAMP test was used to assess Agr activity $[7,10]$. Briefly, overnight culture of a $\beta$ haemolysin producing strain was streaked down the centre of an SBA plate using a cotton swab and the plate was incubated at $37^{\circ} \mathrm{C}$ for $4-6$ hours. Following this, test samples and controls were streaked perpendicularly to it. Plates were incubated at $37^{\circ} \mathrm{C}$ for $12-16$ hours until the haemolysis patterns became clearly visible. Further incubation at $4^{\circ} \mathrm{C}$ was carried out, if necessary, to enhance haemolysis.

\subsection{Reversibility testing}

A total of 39 Agr negative samples were grown in Tryptic Soy Broth (TSB) to stationary phase. Samples were subculture at a 1000-fold dilution into fresh TSB and grown to stationary phase again. Subcultures were carried out a total of 4 times after which serial dilutions were plated on SBA and colonies were screened for haemolysis. Haemolytic colonies were tested for Agr activity as described above.

\subsection{DNA sequencing}

Genomic DNA was purified using standard procedures. The whole agr locus (encompassing hld) was amplified by PCR using the primers agr front and agr back and submitted to direct sequencing (Fasmac, Japan) using the walking primers created for each strain listed in Table 1. The hla locus was amplified using the primers alpha PCR F and alpha PCR R and submitted to direct sequencing (Fasmac, Japan) using the 'alpha' walking primers listed in Table 1. The sequence data were analysed using the DNASTAR sequence analysis suite. 
Table 1. primers used in this study.

\begin{tabular}{c|c}
\hline PRIMER NAME & SEQUENCE $\left(\mathbf{5}^{\prime} \rightarrow \mathbf{3}^{\prime}\right)$ \\
\hline agr front & AGTTGGGATGGCTTAATAAC \\
agr back & CAGCTATACAGTGCATTTGC \\
3010 WPF1 & GAAGACATGCTCATGGTGC \\
3010 WPF2 & GGAATTTCAACATTATCGTTATTTCTA \\
$3010 / 3011 / 3082 / 3114$ WPR1 & CAGTTGCGAGGGCAATTTC \\
$3010 / 3011 / 3082 / 3114$ WPR2 & TCACGTAGGCCAGGCAT \\
3011/3082/3114 WPF1 & GAAGACATGCACATGGTGC \\
$3011 / 3082$ WPF2 & GTAATTTCGACATTATCGTTATTTCTA \\
3011 WPF3 & TGTATGTATCAGGCGTTTC \\
3011 WPR3 & GTACTCTTTAGTCGATTGTGGG \\
3082 WPF3 & \\
3114 WPF2 & GGAATTTCGACATTATCGTTATTTCTA \\
$3114 / 3082$ WPF3 & TGCGAAGACGATCCAA \\
alpha pcr F & CACTCAGTAATTTATCAGTTGC \\
alpha pcr R & CACCTCATATAGTGTCATGTTTAGTC \\
alpha F1 & GATATGTCTCAACTGCAATATTCTA \\
alpha F2 & GTTTAGCCTGGCCTTCAGC \\
alpha F3 & GCAGCAGATAACTTCCTTGAT \\
alpha R1 & TCTGAAGTTATCGGCTAAAGT \\
alpha R2 & GATTGCCATATACCGGGTTCC \\
alpha R3 & GCACCTTCTTCGCTATAAACTC \\
\hline
\end{tabular}

\section{Results and Discussion}

\subsection{9 primary patient samples were characterised as Agr negative}

Primary samples were collected as swabs or liquid blood samples from across the Kanto region of Japan. They were subject to a haemolytic screen on SBA plates to confirm an avirulent phenotype and stocks were made by resuspending single colonies in 20\% glycerol TSB. A CAMP test was used to confirm the Agr negative status of the clinical sample stocks. The test works on the principle of interaction between different haemolysins. Samples are streaked towards a $\beta$-haemolysin producing strain and if the sample produces $\delta$-haemolysin, a direct indicator of Agr activation, an arrowhead pattern of haemolysis emerges from the synergistic effect of $\beta$ and $\delta$ haemolysins. If the sample produces $\beta$-haemolysin, a diffuse pattern of haemolysis is observed. Alpha haemolysin is inhibited by $\beta$-haemolysin, thus if the samples produces $\alpha$-haemolysin an inhibition of haemolysis at the convergence of the two streaks is observed.

\section{2. $10 \%$ of strains reverted haemolysis}

We previously demonstrated that Agr negative variants could revert their Agr system through phase variation. In order to assess the significance of our findings, we carried out the reversibility test with the 39 Agr-negative primary clinical samples. Four out of 39 strains tested phenotypically reverted haemolysis, with one of them reverting its Agr status (Fig. 1). Interestingly, one strain reverted to a phenotype characteristic of $\alpha$-haemolysin production (Fig. 1D). A single strain reverted a $\beta$-haemolysin phenotype (data not shown), but this could likely be due to phage excision from the hlb gene. The Agr loci of the four revertants were sequenced alongside their variant counterparts to identify the genetic mechanism of reversion. However, there were no changes observed for strains 3010, 3011, and 3114. The 3082 revertant had an SNP in the agrC ORF, but this does not correlate to 
any known mechanisms of phase variation and could be a random mutation. Additionally, the hla locus of the 3114 variant and revertant were sequenced, but no changes were observed.

Figure 1 legend $10 \%$ of clinical strains showed phenotypic reversion of haemolysis on a CAMP test. A S. aureus strain MW2 as positive control for Agr activity and its isogenic agr deletion mutant as a negative control. B Strain 3082 reverted to an Agr active phenotype. C Strains 3010 and 3011 reverted a haemolytic phenotype, but Agr activity was unaffected. D Strain 3114 reverted to an $\alpha-$ haemolysin producing phenotype, indicated by the inhibition of haemolysis at the convergence with the Hlb producer but strong haemolysis elsewhere.

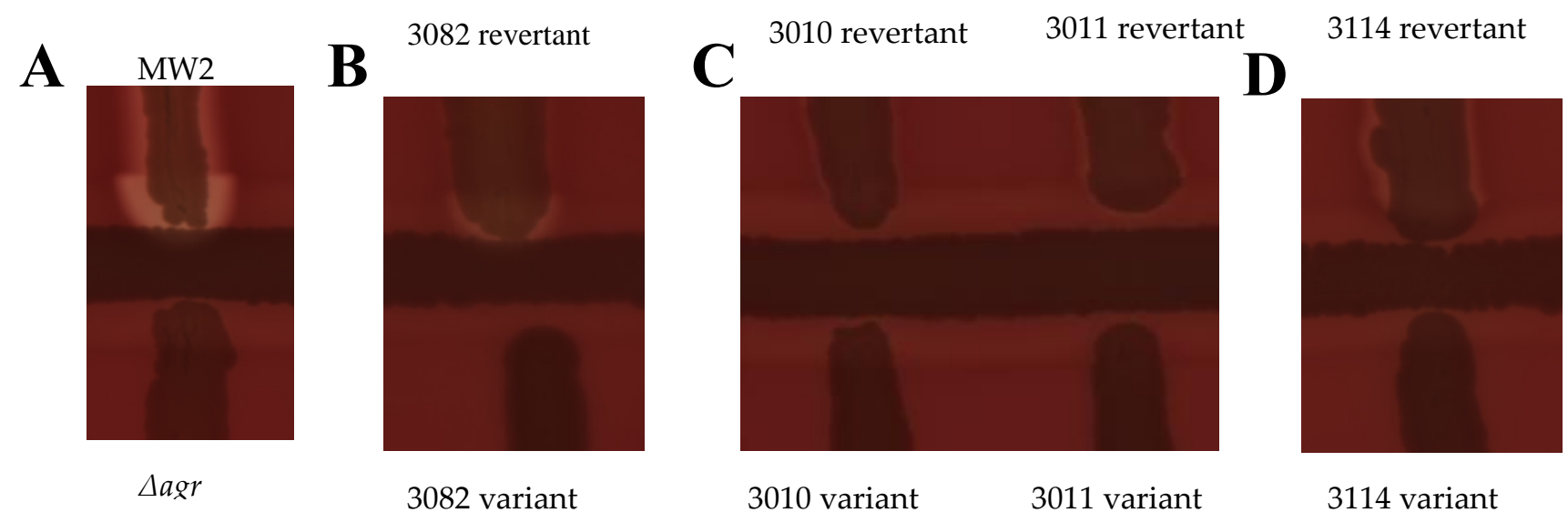

Figure 1. Four clinical strains showed phenotypic reversion of haemolysis.

\section{Conclusion}

In this study, we aimed to investigate the clinical significance of our previous findings of Agr phase variants. Although we could not assess the prevalence of Agr phase variants in the clinical setting, we demonstrated that $10 \%$ of the primary clinical samples used in our study could revert their virulence to some degree. Interestingly, we identified one strain that reverted an Alpha haemolysin phenotype. This data adds a further layer to the complexity surrounding Agr dysfunction amongst clinical S. aureus isolates. Additionally, these results corroborate a recent finding describing how Agr deficient clinical strains develop compensatory mutations during infection to bypass the defects of Agr shutdown [11].

Conflicts of Interest: The authors declare no conflict of interest.

\section{References}

1. Tong, S.Y., et al., Staphylococcus aureus infections: epidemiology, pathophysiology, clinical manifestations, and management. Clin Microbiol Rev, 2015. 28(3): p. 603-61.

2. Tam, K. and V.J. Torres, Staphylococcus aureus Secreted Toxins and Extracellular Enzymes. Microbiol Spectr, 2019. 7(2).

3. Wang, B. and T.W. Muir, Regulation of virulence in Staphylococcus aureus: molecular mechanisms and remaining puzzles. Cell chemical biology, 2016. 23(2): p. 214-224.

4. Bronesky, D., et al., Staphylococcus aureus RNAIII and Its Regulon Link Quorum Sensing, Stress Responses, Metabolic Adaptation, and Regulation of Virulence Gene Expression. Annu Rev Microbiol, 2016. 70: p. 299316. 
5. Fowler, V.G., Jr., et al., Persistent bacteremia due to methicillin-resistant Staphylococcus aureus infection is associated with agr dysfunction and low-level in vitro resistance to thrombin-induced platelet microbicidal protein. J Infect Dis, 2004. 190(6): p. 1140-9.

6. Shopsin, B., et al., Prevalence of agr dysfunction among colonizing Staphylococcus aureus strains. J Infect Dis, 2008. 198(8): p. 1171-4.

7. Traber, K.E., et al., agr function in clinical Staphylococcus aureus isolates. Microbiology, 2008. 154(Pt 8): p. 2265-74.

8. van der Woude, M.W. and A.J. Baumler, Phase and antigenic variation in bacteria. Clin Microbiol Rev, 2004. 17(3): p. 581-611, table of contents.

9. Gor, V., et al., Finding of Agr Phase Variants in Staphylococcus aureus. mBio, 2019. 10(4): p. e00796-19.

10. Christie, K., N. Atkins, and E. Munch-Petersen, A NOTE ON A LYTIC PHENOMENON SHOWN BY GROUP B STREPTOCOCCI. Australian Journal of Experimental Biology and Medical Science, 1944. 22(3): p. 197-200.

11. Altman, D.R., et al., Genome Plasticity of agr-Defective Staphylococcus aureus during Clinical Infection. Infection and Immunity, 2018. 86(10): p. e00331-18.

Publisher's Note: MDPI stays neutral with regard to jurisdictional claims in published maps and institutional affiliations.

(C) 2020 by the authors; licensee MDPI, Basel, Switzerland. This article is an open access article distributed under the terms and conditions of the Creative Commons Attribution (CC-BY) license (http://creativecommons.org/licenses/by/4.0/). 